\title{
Building Fences: How A20 Protects the Intestinal Mucosa in Inflammatory Bowel Diseases
}

\author{
Deenaz Zaidi ${ }^{1,2} \cdot$ Eytan Wine $^{1,2,3,4}$
}

Published online: 25 November 2019

○) Springer Science+Business Media, LLC, part of Springer Nature 2019

Inflammatory bowel diseases (IBD), including Crohn disease (CD) and ulcerative colitis (UC), are chronic diseases with no definite cure found to date. The etiology of IBD is complex, as many factors are associated with disease onset and/or progression, including environmental factors (e.g., geographical variation, smoking, antibiotics, diet, and microbes), genetics, and a dysfunctional gut barrier [1]. Multifactorial involvement renders it difficult to pinpoint a single causative agent and provide targeted treatment for IBD. As a result, current treatment paradigms focus on inducing remission and reducing the frequency of flares, mostly by suppressing the uncontrolled immune response; this of course introduces treatment risks, including infection and malignancy.

The chronic inflammation associated with IBD centers around activation of nuclear factor kappa-light-chainenhancer of activated B cells (NF- $\kappa \beta)$ and tumor necrosis factor- $\alpha$ (TNF- $\alpha)$; indeed, inhibition of TNF- $\alpha$ is an effective treatment for IBD. NF- $\kappa \beta$, an important contributor to innate immunity and altered gut homeostasis in IBD, activates NF- $\kappa \beta$, exacerbating inflammation [2]. TNF- $\alpha$, which is downstream of NF- $\kappa \beta$, is an important regulator of gut barrier function associated with IBD in that it regulates intestinal epithelial cell shedding and is associated with gut

Eytan Wine

wine@ualberta.ca

Deenaz Zaidi

deenaz@ualberta.ca

1 Department of Pediatrics, University of Alberta, Edmonton, $\mathrm{AB}$, Canada

2 Centre of Excellence for Gastrointestinal Inflammation and Immunity Research (CEGIIR), University of Alberta, Edmonton, AB, Canada

3 Department of Physiology, University of Alberta, Edmonton, $\mathrm{AB}$, Canada

4 Division of Pediatric Gastroenterology, ECHA, Room 4-577, 11405 87th Ave, Edmonton, AB T6G 1C9, Canada barrier disruption [3]. Therefore, novel approaches to suppress or better regulate the NF- $\kappa /$ TNF- $\alpha$ pathways carry excellent potential to benefit IBD patients.

The close association of TNF- $\alpha$-induced protein 3 , also known as A20, with NF- $\kappa \beta$, and TNF- $\alpha$ warrants further investigation to better define potential mechanisms for regulating inflammation in IBD. The $A 20$ gene is induced by NF- $\kappa \beta$ activation, whereas A20 protein negatively regulates NF- $\kappa \beta$ signals. Indeed, A20 is involved in controlling inflammation in IBD through multiple mechanisms, such as maintaining the integrity of the gut barrier and controlling microbial influx from the gut lumen to the underlying tissue [4]. Conditional knockout of A20 in intestinal epithelial cells in mice increased apoptosis and influx of commensal bacteria after administration of TNF- $\alpha$ [5]. The gut barrier protective function of A20 is further elucidated by a study in which mice with A20 overexpression did not exhibit signs of increased intestinal paracellular permeability after lipopolysaccharide (LPS) administration [6]. Bacterial influx to the underlying tissue is frequently mediated through $\mathrm{M}$ cells; however, as paracellular permeability is a marker of facilitated bacterial entry from the lumen to submucosal lymphatic tissues, effects of A20 on the barrier might reflect facilitated transmucosal bacterial uptake. A recent study investigated potential reasons for failure of A20 to downregulate inflammation in pediatric $\mathrm{CD}$. While $A 20$ expression in ileal biopsies of pediatric $\mathrm{CD}$ patients was high, the protein level was low. The difference in levels of $A 20$ expression and protein levels could be due to the low expression of ABIN-1, an A20 accessory protein, and instability of the A20 protein due to inhibition of post-translational phosphorylation, which in turn could be a result of low expression of IKK $\beta$ [7]. Nevertheless, the protective potential of A20 in IBD models has been poorly investigated to date.

In this current study by Donghui Chen and colleagues, published in this issue of Digestive Diseases and Sciences [8], the authors explored the potential protective effect of A20 on intestinal paracellular permeability in a mouse 
model of colitis. The study has explored the connection between immunomodulatory pathways triggered by colitis and the anti-inflammatory effects of A20 in this setting. The experimental arm consisted of mice treated with dextran sodium sulfate (DSS) to chemically induce colitis; the control arm of the study included mice that received only tap water. The study was designed to validate ensuing tissue damage after DSS administration by conducting experiments scoping physical and molecular effects of colitis. First, changes in intestinal paracellular permeability were measured by administering oral FITC-dextran. Western blot analysis of tight junction protein zonula occludins-1 (ZO-1) was conducted to document effects on tight junction protein expression after the onset of colitis. To explore changes in the immune pathways, mononuclear cells from the spleen of the mice were extracted and incubated with antibodies to interleukin (IL)-4 and interferon (IFN)- $\gamma$. Gene expression of $\mathrm{T}$ cell immunoglobulin and mucin domain (TIM)1, TIM-4, thrombospondin-1 (TSP-1), IL-4, and IFN- $\gamma$ was determined as well.

Stark differences between the control and experimental groups were observed, indicating a perturbed immune system along with gut barrier damage as a result of colitis. Physical signs of colitis, including bloody diarrhea and weight loss, were observed in the experimental group. As well, colonic histology showed classic signs of inflammation including crypt distortion, the influx of inflammatory cells, a disrupted epithelial layer, and loss of goblet cells from the mucosal and submucosal layers. In contrast, there were no physical signs of inflammation in the mice of the control group; the colonic tissue did not show any abnormality. Intestinal paracellular permeability was also greatly increased in the experimental group (determined by FITC-dextran levels), as compared to the control group. In addition, ZO-1 levels were decreased in the experimental group. Immune responses were altered after the induction of colitis, ameliorated by rectal administration of A20. A Th2 immune response was triggered with the onset of inflammation. While the percentage of CD4 $+\mathrm{IFN}-\gamma+\mathrm{T}$ cells was lower in the experimental group, as compared to the control group, the percentage of CD4 + IL- $4+\mathrm{T}$ cells was higher. The percentage of $\mathrm{CD} 4+\mathrm{IFN}-\gamma+\mathrm{T}$ cells was reduced in the experimental group as compared to the control arm of the study. There was also increased TIM-1 and IL-4 RNA expression in the experimental group, relative to the control group.

The authors reported interesting findings that indicate a possible therapeutic effect of A20 in this colitis model. Upon rectal administration of A20, using a recombinant pVAX1A20 eukaryotic expression vector encapsulated in poly (L-lactide-co-glycolide; PLGA) nanoparticles (Nano-A20), delivered in 5 doses ( 2 prior to DSS and 3 doses over the following weeks), colitis symptoms and intestinal paracellular permeability improved in these mice. CD $4+$ IL- $4+\mathrm{T}$ cell differentiation, and TIM-1 and IL-4 expression (Th2 related co-stimulatory molecules) were reduced, whereas the number of CD4 + IFN- $\gamma+$ T cells was increased with rectal A20. Furthermore, TSP- 1 and IFN- $\gamma$ expression were increased. Based on these results, the authors have suggested a protective function of A20, at least in the setting of DSS colitis in mice. Indeed, this is the first study to test A20 as a potential treatment in the context of an IBD model and does present a potential novel therapeutic approach for IBD.

Nevertheless, it is important to recognize the limitations of this study and additional steps required to translate findings to the human IBD setting. This study is conducted in mice and uses an imperfect IBD model; DSS injures the epithelium with resultant impairment of barrier function and greatly depends on tissue repair (which is relevant to IBD, but is not the result of direct microbial and immune pathways, central to its pathogenesis). Nevertheless, this study does corroborate other aforementioned studies in supporting the protective function of A20 in colitis, and it does highlight relevant immune pathways. Additional steps need to be taken in future studies to determine the specific molecular mechanisms through which A20 acts in the setting of inflammation and to translate findings to the human setting. Protein-protein interactions between A20, TSP-1, and IFN- $\gamma$ need to be explored, as well as levels of related proteins, including TIM-1, IL-4, and TSP-1. The protein levels of the A20 accessory protein ABIN-1 should be assessed pre- and post-induction of colitis, as it is important to understand whether the levels of A20 are affected as a result of reduction in its chaperone proteins. One very important aspect in translational medicine is to validate the results of animal research using in vitro models, human intestinal organoid, and human tissues.

A20 expression in IBD varies with disease phenotype. In adult $\mathrm{CD}$ patients, A20 expression was low, accompanied by a low response to anti-TNF therapy [9]; expression was increased in colonic biopsies of adult UC, but not CD [10]. The cause for this variation, which remains unexplained, necessitates additional research in order to decipher the possibly different actions of A20 in the setting of CD and UC.

In conclusion, although the authors have reported an important function of A20 in the setting of an animal colitis model, the results need to be validated. Some important points to ponder are: Will proactive supplementation with A20 protein prevent the onset of IBD? How do different etiological factors associated with IBD affect A20? What are the molecular mechanisms that could possibly cause the anti-inflammatory action of A20 to differ in CD and UC? Regulation of inflammation is essential for the management of IBD and necessitates targeted identification of pro- and anti-inflammatory factors for optimizing therapeutic goals for IBD. As A20 inhibits inflammation and is a potential 
disease biomarker, it is important to dig deeper into the influence of A20 in IBD. Leveraging on studies such as this one will promote novel therapies, allowing for more appropriate, regulated immune responses, which would counteract, or even prevent, the uncontrolled immune response seen in IBD.

\section{Compliance with Ethical Standards}

Conflict of interest The authors have no relevant conflicts of interest.

\section{References}

1. Piovani D, Danese S, Peyrin-Biroulet L, Nikolopoulos GK, Lytras T, Bonovas S. Environmental risk factors for inflammatory bowel diseases: an umbrella review of meta-analyses. Gastroenterology. 2019;157:647-659.

2. Allen IC, Eden K, Nguyen V, Knight C, Sorrentino D. Noncanonical NF- $\kappa \mathrm{B}$ signaling is elevated in inflammatory bowel disease patients and may be associated with therapeutic response. J Immunol. 2017;198(1 Supplement):197.5.

3. Ślebioda TJ, Kmieć Z. Tumour necrosis factor superfamily members in the pathogenesis of inflammatory bowel disease. Mediat Inflamm. 2014;2014:325129.
4. Zaidi D, Wine E. Regulation of nuclear factor kappa-light-chainenhancer of activated B cells (NF- $\kappa \beta)$ in inflammatory bowel diseases. Front Pediatr. 2018;10:317.

5. Vereecke L, Sze M, Mc Guire C, et al. Enterocyte-specific A20 deficiency sensitizes to tumor necrosis factor-induced toxicity and experimental colitis. J Exp Med. 2010;207:1513-1523.

6. Kolodziej LE, Lodolce JP, Chang JE, et al. TNFAIP3 maintains intestinal barrier function and supports epithelial cell tight junctions. PLoS One. 2011;6:e26352.

7. Zaidi D, Huynh Q, Carroll M, et al. Tumor necrosis factor $\alpha$-induced protein 3 (A20) is dysregulated in pediatric Crohn disease. Clin Exp Gastroenterol. 2018;11:217-231.

8. Chen D, Ma L, Hu T et al. A20 restores impaired intestinal permeability and inhibits Th2 response in mice with colitis. Dig Dis Sci. (Epub ahead of print). https://doi.org/10.1007/s10620-019-05860 -8 .

9. Arsenescu R, Bruno MEC, Rogier EW, et al. Signature biomarkers in Crohn's disease: toward a molecular classification. Mucosal Immunol. 2008;1:399-411.

10. Fernandes P, MacSharry J, Darby T, et al. Differential expression of key regulators of Toll-like receptors in ulcerative colitis and Crohn's disease: a role for Tollip and peroxisome proliferatoractivated receptor gamma? Clin Exp Immunol. 2016;183:358-368.

Publisher's Note Springer Nature remains neutral with regard to jurisdictional claims in published maps and institutional affiliations. 